\title{
Exploring Correlation Between Biofilm Formation Ability and Resistance Potential of Acinetobacter baumannii Strains Isolated from Different Clinical Sources
}

\author{
Nutan Thakur ${ }^{1}$, Chetansee Khanna ${ }^{1}$, Priyanka Sharma ${ }^{2}$, Arti Kapil ${ }^{2}$, Rahul Srivastava ${ }^{1}$, \\ Jitendra Vashistt ${ }^{1 *}$ \\ ${ }^{1}$ Department of Biotechnology \& Bioinformatics, Jaypee University of Information Technology, Waknaghat, Solan, Himachal Pradesh, \\ India-173234 \\ ${ }^{2}$ Department of Microbiology, All India Institute of Medical Sciences, New Delhi, India
}

Address for Correspondence: Jitendra Vashistt, jitendraa.vashistt@juit.ac.in

\begin{abstract}
Keywords Acinetobacter

Baumannii;

Antimicrobial

Regimen;

Nosocomial;

Biofilm.
\end{abstract}

\begin{abstract}
Acinetobacter baumannii belongs to ESKAPE group of pathogens and has emerged as one of the leading causes of nosocomial infections. Biofilm formation is predicted as one of the key pathogenic features of $A$. baumannii, particularly with device-related infections. Moreover, emergence of alarming rates of multidrug-resistance has narrow down the choice of currently available antimicrobials. Present study investigated correlation of multidrug resistance capacity of different $A$. baumannii strains and their biofilm forming ability on polystyrene surfaces. A. baumannii isolates from different clinical sources were collected from Department of Microbiology, AIIMS New Delhi. Antibiotics chosen for AST and MIC experiments were based on their usage as conventional antimicrobial regimen (levofloxacin, tetracycline, amikacin, meropenem, imipenem, ceftazidime, cefepime, piperacillin-tazobactum, cefoperazone-subactum) prescribed for treating $A$. baumannii infections and results were interpreted according to CLSI \& ICMR guidelines. Biofilm forming capacity of each $A$. baumannii isolate was evaluated by qualitative and quantitative methods using microscopy and microtitre-plate-assay, respectively at $37^{\circ} \mathrm{C} \& 44^{\circ} \mathrm{C}$ temperatures for two different time periods i.e. $24 \&$ 48 hours. A. baumannii isolates from urine, pus, respiratory, peritoneal fluid and blood sources exhibited high levels of resistance against all antibiotics in increasing order of magnitude. In medically relevant surface, approximately $50 \%$ of strains were characterized as strong biofilm inducer while half of isolates were found as weak biofilm-inducers following incubation for $24 \mathrm{~h}$. A. baumannii isolates from respiratory, pus, blood and peritoneal fluid showed increased capacity to form biofilm at $37^{\circ} \mathrm{C}$. At $48 \mathrm{~h}$, proportion of strong biofilmformer increased by 1.2 fold. Overall, highest proportion of antibiotic resistant strains from various sources expressed increased ability to form biofilm than susceptible. Recent studies shows disparity in role of antibiotic resistance strains with higher expression of biofilm formation. Biofilm provides opportunity to bacteria to survive in adverse environment and multidrug-resistant nature of pathogens had deteriorated therapeutic options. (c) 2016 iGlobal Research and Publishing Foundation. All rights reserved.
\end{abstract}

Conference Proceedings: International Conference on Advances in Plant and Microbial Biotechnology (PMB2017); JIIT, Noida: February 02-04, 2017

Indo Global Journal of Pharmaceutical Sciences( ISSN 22491023 ; CODEN- IGJPAI; NLM ID: 101610675) indexed and abstracted in EMBASE(Elsevier), SCIRUS(Elsevier),CABI, CAB Abstracts, Chemical Abstract Services(CAS), American Chemical Society(ACS), Index Copernicus, EBSCO, DOAJ, Google Scholar and many more. For further details, visit http://iglobaljournal.com 